\title{
СРАВНЕНИЕ ВАРИАНТОВ КОНСТРУКЦИЙ СИНХРОННЫХ ГЕНЕРАТОРОВ С ВОЗБУЖДЕНИЕМ ОТ ПОСТОЯННЫХ МАГНИТОВ ДЛЯ ВЕТРОУСТАНОВОК
}

\author{
Ю.Н. Перминов ${ }^{1}$, канд.техн.наук, Е.А. Монахов² \\ ${ }^{1}$ Институт возобновляемой энергетики НАН Украины, \\ 02094, г. Киев, ул. Гната Хоткевича, 20A, \\ ${ }^{2}$ Национальный технический университет Украины «Киевский политехнический институт имени Игоря Сикорского», \\ 03059, г. Киев, пр. Победы, 37
}

\begin{abstract}
В статье показана ичелесообразность использования серийных асинхронных двигателей при создании синхронных генераторов с возбуждением от постоянных магнитов. По результатам расчетов даны рекомендации по выбору асинхронных двигателей и соответствующих мультипликаторов при изготовлении синхронных генераторов с возбуждением от высокоэнергетических редкоземельных постоянных магнитов неодим - железо - бор (NdFeB). Применение мультипликатора позволяет повысить частоту вращения генератора, уменьшить его габариты, массу, однако это вызывает увеличение общей массы установки за счет применения мультипликатора. Серийные изделия отличаются оптимальными геометрическими соотношениями, минимизированы по массе, отличаются качеством изготовления, и, следовательно, - надежностью. В работе предложена оригинальная конструкция бескорпусного, безредукторного синхронного магнитоэлектрического генератора для ветроустановок и приведены некоторые результаты расчетов ряда генераторов на базе этой конструкиии мощностью от 20 кBт до $2 \mathrm{MBm}$, а также дана оценка характера изменения геометрических размеров зубиовой зоны статоров в этих генераторах, которая позволяет оценить технологичность их изготовления. Для снижения массогабаритных показателей был предложен, разработан, изготовлен и испытан бескорпусной безредукторный синхронный генератор с возбуждением от постоянных магнитов мощностью 20 кВт. Также в работе представлены некоторые результаты расчетов редукторной и безредукторной компоновки ветроустановки для их последующей оченки, учитывая габариты и массу активных материалов, в том числе редкоземельных постоянных магнитов, иена которых является наибольшей. Библ. 9, табл. 2, рис. 3.
\end{abstract}

Ключевые слова: асинхронные двигатели; синхронные генераторы; мультипликаторы; бескорпусной; постоянные магнитыз; ветроустановка.

\section{DESIGN COMPARISON OF SYNCHRONOUS PERMANENT MAGNET GENERATOR FOR WIND TURBINES}

\author{
Y. Permynov ${ }^{1}$, candidate of technical sciences, E. Monakhov ${ }^{2}$ \\ ${ }^{1}$ Institute of Renewable Energy, NAS of Ukraine, \\ Hnata Khotkevicha Str., 20A, 02094, Kyiv-94, Ukraine \\ ${ }^{2}$ National Technical University of Ukraine «Igor Sikorsky Kyiv Polytechnic Institute» \\ Peremohy av., 37, 03059, Kyiv-59, Ukraine
}

The article points out the expediency of usage the asynchronous motor for re-design them into synchronous generator with permanent magnet excitation. According to the calculation results there are given some recommendations in questions such as choice of the asynchronous motor and its corresponding speed multiplier for manufacturing synchronous generator with excitation of high-energy rare-earth permanent magnets neodymium - iron - boron (NdFeB). Application of speed multiplier allow to increase rotation speed of generator, decrease its dimensions and mass, but it causes increasing the general mass of set due to installation of speed multiplier. Serial industrial products have optimal geometrical ratios, minimized by weight, have high quality of manufacturing and, consequently - are reliable. An original design of caseless gearless synchronous permanent magnet generator for wind turbine is presented and a number of calculations in a range of power since $20 \mathrm{~kW}$ till $2 \mathrm{MW}$ is provided. An evaluation of geometric changes in tooth zone of stator in these generators are given that allows to estimate its manufacturability. For declining the weight and size indicators the caseless gearless synchronous permanent magnet generator was proposed, designed, made and tested. The power of generator is $20 \mathrm{~kW}$. Also there are given some results of calculation of gear and gearless windturbine for its further estimation, taking into account its dimensions and mass of active materials, including rare-earth permanent magnets, the price of which is the most. References 9, tabl. 2, figures 3 .

Keywords: asynchronous motor, synchronous generator, speed multiplier, caseless, permanent magnet, wind turbine.

(C) Ю.Н. Перминов, Е.А. Монахов, 2019

Відновлювана енергетика. 2019. № 2 


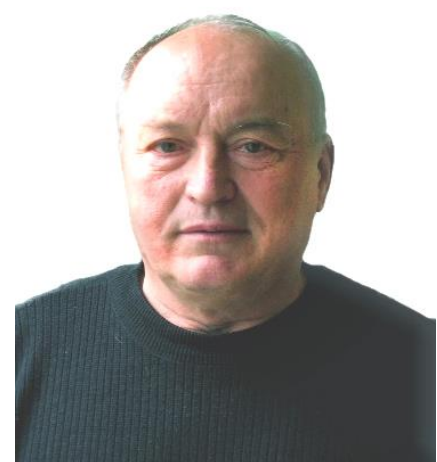

Ю.Н. Перминов

Y. Permynov

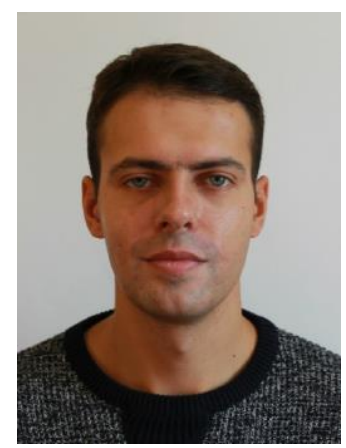

E.A. Монахов

E. Monakhov
Сведения об авторе: канд. техн. наук, старший научный сотрудник Института возобновляемой энергетики НАН Украины. Образование: окончил в 1968 г. Киевский политехнический институт по специальности «Электрические машины и аппараты». Область научных интересов: ветрогенераторы, ветроустановки малой мощности, гидрогенераторы, устройства с постоянными магнитами.

Публікації: 85 .

ORCID: $10000-0001-5604-8327$

Контакты: Тел./факс +38-044-206-28-09

e-mail: renewable@ukr.net

Сведения об авторе: асистент кафедры электромеханики КПИ им. И. Сикорского, Киев.

Образование: окончил в 2012 г. Киевский политехнический институт по специальности «Электрические машины и аппараты». Научная сфера: машины с постоянными магнитами, синхронные генераторы, малые электростанции, гидроэлектростанции.

Публикации: 24

ORCID: 0000-0001-8408-8051

Контакты: тел./факс: +38-095-317-61-15

e-mail: emonachov@gmail.com
Author information: candidate of technical sciences, Senior Researcher at Institute for Renewable Energy, National Academy of Sciences of Ukraine.

Education: graduated from the Kiev Polytechnic Institute in 1968 with a degree in Electrical Machines and Apparatuses.

Research area: wind power systems, small capacity wind units, hydrogenerators, devices with permanent magnets

Publications: 85 .

ORCID: $10000-0001-5604-8327$

Contacts: phone./fax: +38-044-206-28-09

e-mail: renewable@ukr.net

Author information: assistant of department of electomechanics in Igor Sikorsky Kyiv Polytechnic Institute.

Education: graduated from the Kyiv Polytechnic Institute in 2012 with the specialization «Electrical machines and apparatus».

Research area: permanent magnet machines, synchronous generators, small energy plants, hydropower plants.

Publications: 24.

ORCID: 0000-0001-8408-8051

Contacts: phone./fax: +38-095-317-61-15

e-mail: emonachov@gmail.com

\section{Перечень используемых обозначений:}

$M_{\text {э }} \quad$ - электромагнитный момент;

$P \quad-$ мощность генератора;

$A-$ линейная нагрузка;

$B_{\delta} \quad-$ индукция в рабочем зазоре;

$D_{a} \quad$ - диаметр ротора;

$l_{a} \quad$ - активная длина ротора;

$\rho-$ плотность воздуха;

Введение. В ветроустановках малой мощности широко используются синхронные генераторы с возбуждением от постоянных магнитов, применение которых позволяет упростить конструкцию, исключить потери на возбуждение, существующие в машинах с электромагнитным возбуждением, повысить К.П.Д. и надежность. Поэтому в последние годы магниты применяются не только в машинах малой мощности, но и имеющих мощность сотни кВт. Это обусловливает необходимость решения задач, связанных с проектированием и изготовлением таких машин.

Постановка задачи. В статье рассмотрены возможные решения задачи создания синхронных генераторов с возбуждением от постоянных магнитов для ветроустановок. Один из основных вопросов, которые возникают при проектировании генераторов для ветроустановок, - выполнять их с мультипликаторами, или без них. Электромагнитный момент генератора определяется

$\begin{array}{ll}v & - \text { скорость воздушного потока; } \\ k & - \text { К.П.Д. ветроколеса; } \\ D & - \text { диаметр ветроколеса; } \\ \varpi & - \text { угловая частота вращения; } \\ z^{\prime} & - \text { коэффициент быстроходности; } \\ S & - \text { площадь ометаемой поверхности; } \\ f & \quad-\text { частота напряжения. }\end{array}$

отношением мощности генератора к угловой частоте вращения

$$
M_{\ni}=\frac{P}{\varpi}
$$

Очевидно, что при увеличении частоты вращения и заданной мощности электромагнитный момент уменьшается.

Электромагнитный момент определяется произведением объема ротора на удельные электромагнитные нагрузки

$$
M_{\ni}=\alpha_{i} \cdot\left(A \cdot B_{\delta}\right) \cdot \pi \cdot D_{a}^{2} \cdot l_{a}
$$

где $A=\frac{I_{u} \cdot N}{\pi \cdot D_{a}}-$ линейная нагрузка $(\mathrm{A} / \mathrm{M})$

$I_{\mu}$ - номинальный ток; $N$ - число проводников в обмотке; $B_{\delta}-$ индукция в рабочем зазоре(Тл);

$$
\pi \cdot D_{a}^{2} \cdot l_{a} \text { - объем ротора, }
$$


где $D_{a}$ - диаметр ротора (м); $l_{a}$ - активная длина ротора (м).

Т. е. с увеличением частоты вращения масса и габариты ротора, и машины в целом - уменьшаются. Отличительная особенность генераторов для ветроустановок - низкая частота вращения, обусловленная геометрией самой установки.

Мощность генератора определяется мощностью ветрового потока

$$
P=\frac{\rho \cdot S \cdot v^{3} \cdot k}{2}
$$

при этом площадь ометаемой поверхности,

$$
S=\frac{2 \cdot P}{\rho \cdot v^{3} \cdot k}
$$

где $\rho=1,3$ кг $/ \mathrm{M}^{3}$ - плотность воздуха; $v$ - скорость ветра $(\mathrm{m} / \mathrm{c}) ; k \approx 0,38 \div 0,4-$ КПД ветроколеса.

Диаметр ветроколеса

$$
D=\sqrt{\frac{S}{0,785}} \text { (м) }
$$

Частота вращения ветроколеса

$$
\varpi=\frac{z^{\prime} \cdot v}{R} \mathrm{pad} / \mathrm{c}
$$

где $z^{\prime}$ - коэффициент быстроходности осевого ветроколеса.

Как видно из (6), (7), (8) с ростом площади ометаемой поверхности, увеличивается мощность установки, диаметр ветроколеса и при заданной скорости ветра уменьшается частота его вращения.

В связи с этим при одинаковой скорости ветрового потока, например, -8 м/с генератор мощностью 20 кВт имеет частоту вращения 100 об/мин, а генератор мощностью 600 кВт 14 об/мин, т.е., примерно, в 800 раз меньше, чем в авиационных генераторах. Это обусловливает большие габариты, массу, цену устройства.

Применение мультипликатора позволяет повысить частоту вращения генератора, уменьшить его габариты, массу, но увеличиваются эти параметры за счет мультипликатора, появляется необходимость его обслуживания, но и возможность изготовления агрегата на базе серийных асинхронных двигателей и мультипликаторов, которых в Украине - достаточно. Серийные изделия отличаются оптимальными геометрическими соотношениями, минимизированы по массе, отличаются качеством изготовления, и, следовательно, - надежностью. Переделка серийного асинхронного двигателя в синхронный генератор с возбуждением от постоянных магнитов не является сложной задачей и заключается только в переделке его ротора. Для получения тех пара- метров генераторы, которые указаны на двигателе необходимо выполнить два условия: обеспечить указанную частоту вращения и индукцию в рабочем зазоре машины, примерно $0,72-0,8$ Тл, как в серийных асинхронных двигателях. Частота вращения обеспечивается необходимым по передаточному числу мультипликатором, а указанная индукция - применением магнитов $\mathrm{NdFeB}$ при несложной переделке ротора машины (рис. 1): шихтованный пакет с беличьей клеткой заменяется стальной болванкой из магнитомягкой стали с гранями, количество и размер которых соответствует числу полюсов (магнитов). Число полюсов определяется по указанной частоте вращения двигателя:

$$
p=\frac{f \cdot 60}{n}
$$

где $f=(50 \div 60)$ Гц - частота напряжения сети; $n$ - частота вращения двигателя (об/мин); $p-$ число пар полюсов.

Конструкция ротора может быть существенно упрощена при применении магнитов подковообразной формы с отверстиями. В этом случае отпадает необходимость фрезеровки граней на болванке и изготовлении полюсных башмаков (рис. 1). В серийных асинхронных двигателях обмотка статора располагается в пазах, в этом случае ограничением величины индукции в рабочем зазоре является насыщение зубцовой зоны, поэтому возможно применение простой магнитной системы с радиальным расположением полюсов чередующейся полярности.

При изготовлении генератора целесообразно использовать серийных двигатель необходимой мощности с минимально возможной частотой вращения - $(750-1000)$ об/мин, т.к. эти машины являются многополюсными - $(8-6)$ полюсов соответственно частоте вращения. Машины с большим числом полюсов имеют меньшую толщину спинки статора по условию насыщения, поэтому меньше её масса, которая составляет до $25 \%$ от общей массы двигателя. Однако при низкой частоте вращения требуется мультипликатор с большим передаточным числом, увеличивается его масса. Таким образом с ростом передаточного числа мультипликатора уменьшается масса требуемого двигателя, но увеличивается масса мультипликатора. Масса двигателя и масса мультипликатора приблизительно пропорциональны диаметрам корпусов машин, в результате минимальная масса агрегата - двигатель - мультипликатор будет при определенном передаточном числе мультипликатора и соизмеримых диаметpax корпусов машин. 


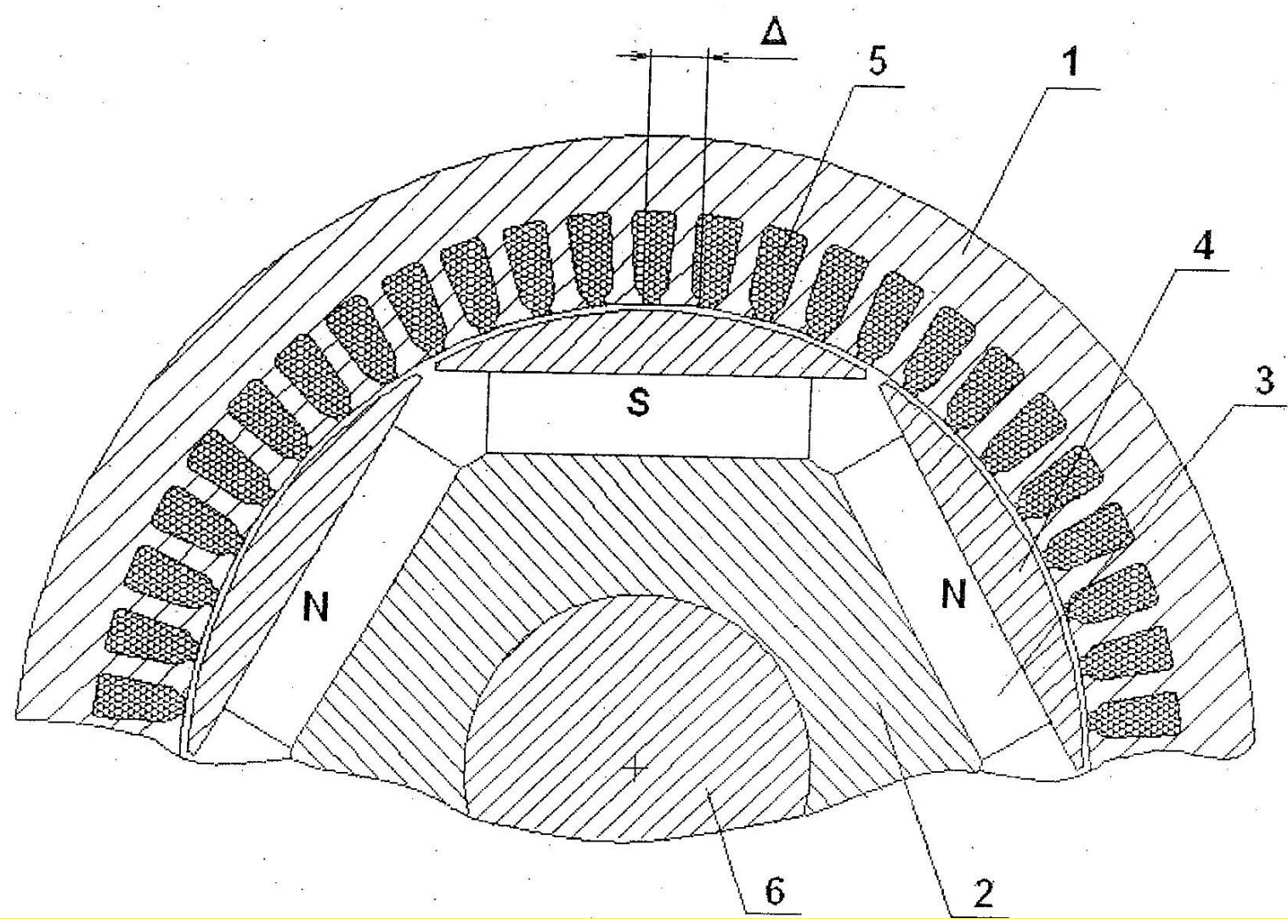

Рис. 1. Конструкция магнитоэлектрического генератора: 1 -cmaтор; 2 - ротор; 3 - постоянные магниты; 4 - полюсные наконечники; 5 -обмотка; 6-вал.

Fig. 1. Design of permanent magnet generator: 1 -stator; 2 -rotor; 3 - permanent magnets; 4 -pole shoes; 5 - winging; 6 - shaft.

Другая постановка задачи проектирования синхронных генераторов с возбуждением от постоянных магнитов заключается в создании безредукторного генератора, т.к. в этом случае упрощается конструкция агрегата в целом и отпадает необходимость обслуживания редуктора (мультипликатора). При решении этой задачи могут быть использованы два подхода: создание генератора на базе серийного асинхронного двигателя; создание генератора оригинальной конструкции. В обеих случаях предполагается наличие производственной цепочки оборудования, аналогичной применяемому на электротехнических заводах и соответствующих технологий, в противном случае это - затратный и растянутый во времени путь не гарантирующих качества продукции. В Украине достаточно таких предприятий, расположены они в Полтаве, Харькове, Днепре, Каховке. Выпускаются машины малой мощности (Полтава), машины широкого диапазона мощностей (Харьков, Каховка). Причем в Каховке есть завод «Крупных электрических машин», который изготавливает машины для «Газпрома», «Лукойла» мощностью до 4000 кВт; в настоящее время на этом заводе идет изготовление оригинальной, безредукторной ветроустановки, в которой используется три синхронных генератора с возбуждением от постоянных магнитов мощностью по 500 кВт каждый. Таким образом, в Украине есть электротехнические заводы, на которых можно изготавливать синхронные генераторы для ветроустановок мощностью от десятков Ватт до нескольких МВт. При создании безредукторного генератора на базе серийного двигателя выбор соответствующей машины осуществляется по требуемому электромагнитному моменту (1), исходя из заданной мощности генератора и угловой частоте вращения ветроколеса (7).

В институте ИВЭ были спроектированы и изготовлены два генератора соизмеримой мощности с возбуждением от постоянных магнитов NdFeB: на базе серийного асинхронного двигателя 4АМУ160М6 («Южэлектромаш», Каховка), серийного мультипликатора с передаточным числом - 9,9 и синхронный генератор без мультипликатора оригинальной конструкции, бескорпусной, где корпусом является шихтованный пакет статора (рис.2) [1]. 


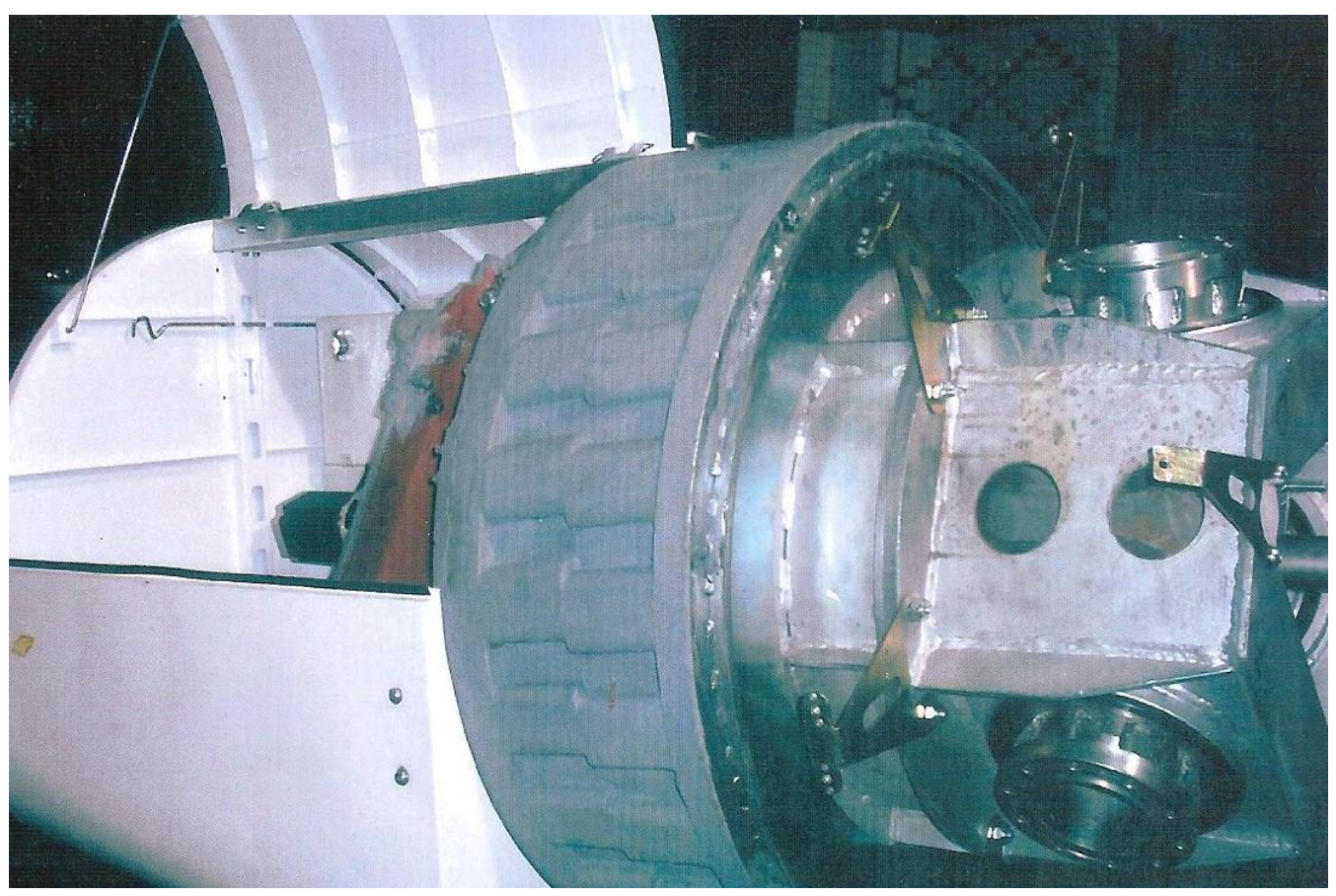

Рис. 2. Общий вид бескорпусного генератора с возбуждением от постоянных магнитов.

Fig. 2. General view of permanent magnet caseless generator.

Генераторы имеют следующие параметры: генератор на базе двигателя 4АМУ160М6 - номинальная мощность $-\mathrm{P}_{\text {н }}=15$ кВт; частота вращения $-n_{н}=1000$ об/мин; номинальный ток $I_{\mu}=29,6 / 51,4$ А; напряжение $-U_{u}=380 / 220 \mathrm{~B}$; К.П.Д. $=88,5 \%$ (на рис. 1 показан ротор этого генератора); и безредукторный бескорпусной генератор (рис. 2) - номинальная мощность $P_{u}=20 \mathrm{\kappa BT;} U_{\phi}=220 \mathrm{~B} ;$ частота вращения - $n_{\mu}=100$ oб/мин; частота выходного напряжения - $f=50$ Гц. Бескорпусная конструкция позволяет уменьшить массу генератора, упростить его изготовление, снизить цену в сравнение с корпусным вариантом.

Данный генератор был использован в ветроустановке соответствующей мощности, которая прошла испытания в течении $3 \mathrm{x}$ лет в Киевской области (рис. 3).

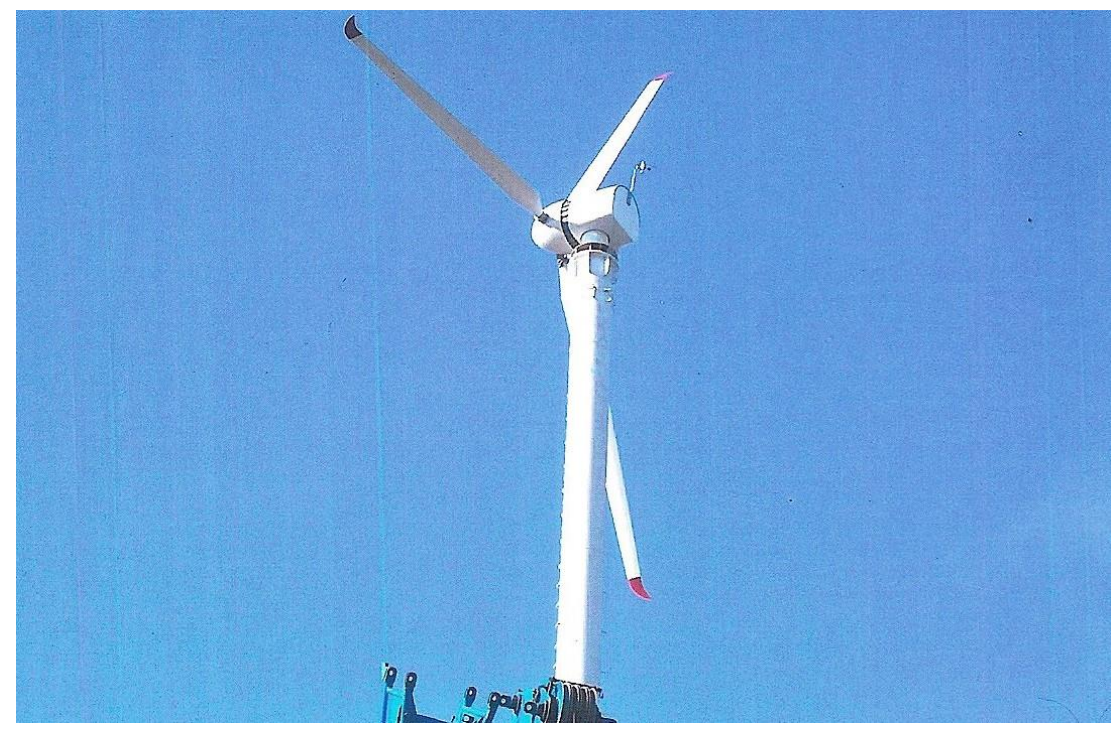

Рис. 3. Экспериментальная ветроустановка с бескорпусным генератором.

Fig. 3. Experimental wind generator with caseless generator.

Близость мощностей генераторов (15 кВт) для агрегата с мультипликатором и без него -
20 кВт позволяет сделать сравнительную оценку их параметров (табл. 1). 
Табл. 1. Параметры синхронных генераторов с возбуждением от постоянных магнитов на базе серийных асинхронных двигателей и синхронных генераторов оригинальной конструкции.

Tabl. 1. Parameters synchronons generators with excitation from PM on the base synchronons generators original construction.

\begin{tabular}{|c|c|c|c|c|c|c|c|c|c|c|c|c|c|c|}
\hline \multirow[t]{2}{*}{ Тип генератора } & $P_{u}$ & $U_{\phi}$ & $I_{\phi}$ & $n$ & $n_{\phi}$ & $f$ & $m_{2}$ & $m_{M}$ & $D_{\kappa n}$ & $L_{\kappa n}$ & $D_{\kappa M}$ & $L_{\kappa M}$ & $k$ & $v$ \\
\hline & $\kappa B m$ & $B$ & $A$ & об/ $/{ }_{\text {мин }}$ & & $\Gamma u$ & $\kappa 2$ & $\kappa 2$ & $M M$ & $\mathcal{M M}$ & $\mathcal{M M}$ & $M M$ & & $M / c$ \\
\hline $\begin{array}{c}\text { На базе серийно- } \\
\text { го асинхронного } \\
\text { двигателя } \\
\text { 4АМУ160М6 }\end{array}$ & 15 & 220 & 29,6 & 1000 & 3 & 50 & 160 & 100 & 340 & 590 & 350 & 360 & 9,9 & 8 \\
\hline $\begin{array}{c}\text { Бескопрусной, } \\
\text { без мультипли- } \\
\text { катора }\end{array}$ & 20 & 220 & 34,53 & 100 & 3 & 30 & 289 & - & 845 & 300 & - & - & - & 8 \\
\hline
\end{tabular}

В таблице 1 приняты следующие обозначения: $P_{H^{-}}$номинальная мощность; $U_{\phi}-$ фазное напряжение; $I_{\phi}$ - фазный ток; $n$ - частота вращения; $n_{\phi}$ - число фаз; $m_{\text {г }}$ - масса генератора; $m_{\mu}$ - масса мультипликатора; $D_{k n}$ - диаметр корпуса генератора; $L_{\kappa n}$ - длина корпуса генератора; $D_{\kappa м}$ - диаметр корпуса мультипликатора; $L_{\kappa м}$ - длина корпуса мультипликатора; $k$ - передаточное число мультипликатора; $v$ - скорость ветра.

Из табл. 1 следует, что при соизмеримых мощностях суммарная масса генератора с мультипликатором сопоставима с массой бескорпусного генератора без мультипликатора, но геомет- рические размеры существенно отличаются: в первом случае - диаметр генератора составляет 340 мм; диаметр мультипликатора - 350 мм; суммарный осевой размер генератора и мультипликатора - 950 мм; во втором случае - диаметр корпуса - 845 мм, длина корпуса - 300 мм, т.е. осевой размер генератора существенно меньше.

Приняв за основу бескорпусную машину, без мультипликатора был проведен расчет ряда таких генераторов мощностью от 20 кВт до 2 МВт при скорости ветра -8 м/с, ориентируясь на наиболее активные ветрозоны Украины $(4,5-5$ м/с). Некоторые результаты расчетов приведены в табл. 2.

Табл. 2. Расчеты синхронных генераторов оригинальной конструкции мощностью от 20 кВт до 2 мВт.

Tabl. 2. An original desing of caseless synchronons generators is presented and the numbers of calculation in a range of power from $20 \mathrm{~kW}$ to $2 \mathrm{~mW}$ is provided.

\begin{tabular}{|c|c|c|c|c|c|c|c|c|c|c|c|c|}
\hline$P_{\mu}$ & $U_{\phi}$ & $n$ & $f$ & $D_{\kappa n}$ & $D_{p}$ & $L_{p}$ & $L_{\kappa n}$ & $m_{n м}$ & $m_{2}$ & $b_{z}$ & $h_{n}$ & $b_{u}$ \\
\hline$\kappa B m$ & $B$ & $o \sigma / \mathcal{M \mu}$ & $\Gamma u$ & $\mathcal{M M}$ & $\mathcal{M M}$ & $\mathcal{M M}$ & $\mathcal{M M}$ & $\kappa 2$ & $\kappa 2$ & $\mathcal{M M}$ & $\mathcal{M M}$ & $\mathcal{M M}$ \\
& & & & & & & & & & & \\
\hline 20 & 220 & 100 & 30 & 835 & 700 & 200 & 300 & 29 & 289 & 10 & 25 & 3 \\
\hline 50 & 220 & 50 & 50 & 1300 & 1200 & 350 & 454 & 99 & 650 & 6 & 28 & 3,5 \\
\hline 120 & 220 & 32 & 50 & 1850 & 1700 & 520 & 616 & 215 & 1543 & 6 & 45 & 3 \\
\hline 600 & 690 & 14 & 50 & 3500 & 3200 & 980 & 976 & 772 & 7731 & 6 & 56 & 3 \\
\hline 2000 & 690 & 8 & 50 & 4700 & 4400 & 1400 & 1450 & 1712 & 21000 & 6,3 & 75 & 6 \\
\hline
\end{tabular}

В таблице 2 приняты следующие обозначения: $D_{p}$ - диаметр ротора; $L_{p}$ - длина ротора; $m_{n м}$ - масса постоянных магнитов; $b_{z}$ - ширина зубца статора; $h_{n}$ - глубина паза; $b_{u}-$ ширина щели между зубцами.

Как указывалось, увеличение мощности установки сопровождается уменьшением частоты вращения и при заданной частоте выходного напряжения увеличивается число полюсов ротора (8). Поэтому при принятом числе пазов на полюс и фазу, например - q = 1 происходит увеличение глубины пазов, а ширина зубцов - невелика (табл. 2). Это может обусловить технологические трудности изготовления пакета статора. Увеличение мощности генератора при заданном напряжении сопровождается ростом тока и сечения провода, уменьшением числа витков в секции и необходимостью выполнения обмотки с несколькими параллельным ветвями.

При проведении расчетов ряда ветрогенераторов (табл. 2) использовались работы [2-9].

Выводы. 1. Проведена оценка зависимости параметров ветроустановки, генератора от скорости ветра.

2. Показана целесообразность использования серийных асинхронных двигателей при создании синхронных генераторов с возбуждением от постоянных магнитов для ветроустановок.

3. Даны рекомендации по выбору асинхронных двигателей и мультиприкаторов при созда- 
нии синхронных генераторов с возбуждением от постоянных магнитов.

4. Дана рекомендация по выбору асинхронного двигателя при создании на его базе безредукторного синхронного генератора.

5. Определена оригинальная конструкция безредукторного бескорпусного синхронного генератора для ветроустановки.

6. Приведены некоторые результаты расчетов ряда безредукторных, бескорпусных генераторов с возбуждением от постоянных магнитов мощностью от 20 кВт до 2 МВт, дана оценка характера изменения геометрических размеров зубцовой зоны статоров в этих генераторах.

1. Кудря С.О., Перминов Ю.М., Коханевич В.П. Патент на винахід № 106673. Україна. МПК Н02K 16/00. Н02К 21/22. Вітроелектрична установка модульної конструкції. № a 2013 01473. заял. 07.02.13. опубл. 25.09.14, Бюл. №9.

2. Перминов Ю.Н., Конахевич В.П., Шихайлов Н.А., Перминова С.Ю. Определение параметров и основных размеров генераторов для приливных электростанций малой мощности (до 1000 кВт). Відновлювальна енергетика. 2017 №3. C. 66-72.

3. Кудря С.А., Перминов Ю.М. Оптимизация системы возбуждения электрических машин. Возобновляемая энергетика. 2012. №4. С. 57-62.

4. Кудря С.А., Перминов Ю.Н., Будьонный В.Ф. Дослідження магнітних систем збудження синхронних вітрогенераторів. Відновлювальна енергетика. 2007. №2. С. 55-59.

5. Перминов Ю.Н., Коханєвич В.В., Монахов С.А. Разработка методики расчета теплогенератора с постоянным магнитами. Відновлювальна енергетика. 2017. №1. С.56-61.

6. Кудря С.А., Перминов Ю.Н. О методах расчета магнитных систем с постоянными магнитами. Відновлювальна енергетика. 2009. №4. С. 40-43.

7. Балагуров В.А., Галтеев Ф.Ф., Ларионов А.Н. Электрические машины с постоянными магнитами. М. Энергия. 1964. $208 \mathrm{c}$.

8. Арнольд P.P. Расчет и проектирование магнитных систем с постоянными магнитами. М. Энергия. 1969. 260 с.

9. Буль Б.К. Основы теории и расчета магнитных цепей. М. Энергия 1964. 154 с.

\section{REFERENCES}

1. Kudrya S.O., Perminov Yu.M., Kokhanevich V.P. Patent Ukraine № 106673. IPC H02K 16/00. H02K 21/22. Windelectric modular set. № a 2013 01473. stated. 07.02.13. published 25.09.14. Bul. № 9. [in Ukrainian].

2. Perminov Yu., Kohanevich V., Shihaylov N., Perminova $S$. Opredelenie parametrov i osnovnyih razmerov generatorov dlya prilivnyih elektrostantsiy maloy moschnosti (do $1000 \mathrm{kVt}$ ). [Determination of parameters and basic dimensions of the generator of low power tidal plants (up to $1000 \mathrm{~kW}$ )]. Vidnovluvana energetika. 2017. No.3. Pp. 66-72. [in Russian].

3. Kudrya S., Perminov $Y u$. Optimizaciya system vozbuzhdeniya elektricheskikh maschin. [The optimization of excitation system of electric machines]. Vozobnovlyaemaya energetika. [Renewable energy]. 2012. No. 3. Pp. 57-62. [in Russian].

4. Kudrya S., Perminov Yu., Budioniy V. Doslidzhenya magnitnikh system zbudzhennya sinkhronikh vitrogeneratoriv. [The research on magnetic excitation systems of synchronous windgenerator]. Vidnovluvana energetika. 2007. No. 2. Pp. 5559. [in Russian].

5. Perminov Yu., Kohanevich V., Monakhov E. Razrabotka metodiki rascheta teplogeneratora $\mathrm{s}$ postoyanimi magnitami. [The development of design calculation methodic of heatgenerator with permanent magnets]. Vidnovluvana Energetyka. 2017. No.1. Pp. 56-61. [in Russian].

6. Kudrya S., Perminov Yu. O metodakh rascheta magnitnikh system s postoyanimi magnitami. [About calculation mathods of the magnetic systems with permanent magnets]. Vidnovluvana Energetika. 2009 No.4. Pp. 40-43. [in Russian].

7. Balagurov V., Galteev F., Larionov A. Elekticheskie mashini s postoyanimi magnitami. [Permanent magnet machines]. Moscow. Russia. Eneriya. 1964. 208 p. [in Russian].

8. Arnold $R$. Rashet i proektirovanie magnitnikh system s postoyanimi magnitami. [Calculation and design the magnetic systems with permanent magnets]. Moscow. Russia. Energiya. 1969. 260 p. [in Russian].

9. Bul B. Osnovi teorii I rascheta magnitnikh cepej. [Basics of theory and calculation of magnetic circuits]. Moscow. Russia. Energiya. 1964. 154 p. [in Russian].

\section{ПОРІВНЯННЯ ВАРІАНТІВ КОНСТРУКЦІЙ СИНХРОННИХ ГЕНЕРАТОРІВ ЗІ ЗБУДЖЕННЯМ ВІД ПОСТІЙНИХ МАГНІТІВ ДЛЯ ВІТРОУСТАНОВОК}

\section{Ю.Н. Перминов ${ }^{1}$, канд.техн.наук, С.А. Монахов ${ }^{2}$}

${ }^{1}$ Інститут відновлюваної енергетики НАН України, 02094, м. Киев, вул. Гната Хоткевича, 20А

${ }^{2}$ Національний технічний університет України «Київський політехнічний інститут імені Ігоря Сікорського», 03059, м. Киев, пр. Перемоги, 37

У статті показана доцільність використання серійних асинхронних двигунів при створенні синхронних генераторів зі збудженням від постійних. По результатам розрахунків надані рекомендації щзодо вибору асинхронних двигунів та відповідних мультиплікаторів при виготовленні синхронних генераторів зі збудженням від високоенергетичних рідкоземельних постійних магнітів неодим - залізо - бор (NdFeB). Застосування мультиплікатора дозволяс підвищити частоту обертання генератора, зменшити його габарити, масу, однак ие викликає збільшення загальної маси установ-

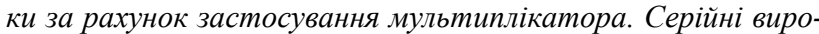
би мають оптимальні геометричні співвідношення, мінімізовані по масі, мають високу якість виготовлення, $i$, відповідно - надійність. У роботі запропонована оригінальна конструкція бескорпусного безредукторного синхронного магнітоелектричного генератора для вітроустановок та приведені деякі результати розрахунків ряду генераторів на базі иієї конструкиї потужністю від 20 кBm до $2 \mathrm{MBm}$, а також надана оцінка характеру зміні геометричних розмірів зубиевої зони статорів цих генераторів, котра дозволяє оиінювати технологічність їх виконання. Для зниження масо-габаритних показників був запропонований, розроблений, виготовлений та випробуваний бескорпусной безредукторний синхронний генератор зі збудженням від постійних магнітів потужністю 20 кВт. Також в роботі представлені деякі результати розрахунків редукторної і безредуктроної компоновки вітроустановки для їх подальшої оцінки, враховуючи габарити та масу активних матеріалів, в тому числі рідкоземельних постійних магнітів, иіна котрих є найбільшою. Бібл. 9, табл.. 2, рис. 3.

Ключові слова: асинхронні двигуни; синхронні генератори; мультиплікатори; бескорпусний; постійні магніти; вітроустановки. 